\title{
Erratum: Depleting endogenous proteins
}

Ada Yee

Nat. Methods 15, 17 (2018); published online 3 Jan 2018; corrected after print 7 February 2018

In the version of this article initially published, the affiliation of Leo James was incorrectly stated as Cambridge University; the correct affiliation is MRC Laboratory of Molecular Biology, Cambridge. Furthermore, the antibody receptor described was incorrectly called TRIM2; the correct name is TRIM21. The errors have been corrected in the HTML and PDF versions of the article.

\section{Corrigendum: Automated structure modeling of large protein assemblies using crosslinks as distance restraints}

\author{
Mathias Ferber, Jan Kosinski, Alessandro Ori, Umar J Rashid, María Moreno-Morcillo, Bernd Simon, Guillaume Bouvier, \\ Paulo Ricardo Batista, Christoph W Müller, Martin Beck \& Michael Nilges \\ Nat. Methods 13, 515-520 (2016); published online 25 April 2016; corrected after print 7 February 2018 \\ In the version of this article initially published, an important funding source, the Agence National de Recherche (ANR-10-BINF-0003 \\ BIP:BIP to M.N.), was omitted. The error has been corrected in the HTML and PDF versions of the article.

\section{Corrigendum: Nm-seq maps 2'-0-methylation sites in human mRNA with base precision}

Qing Dai, Sharon Moshitch-Moshkovitz, Dali Han, Nitzan Kol, Ninette Amariglio, Gideon Rechavi, Dan Dominissini \& Chuan He Nat. Methods 14, 695-698 (2017); published online 15 May 2017; corrected after print 28 February 2018

We were alerted by readers that the reported $\mathrm{Nm}$ consensus sequence in mRNA matches the $3^{\prime}$-adaptor sequence used in sequencing library preparations, and this could be caused by mispriming ${ }^{1}$. In our approach, the majority of RNA fragments without $\mathrm{Nm}$ at the $3^{\prime}$ end are blocked from ligating to the $3^{\prime}$ adaptor because of the presence of $3^{\prime}$ phosphate from the last oxidation elimination step (OE) (Fig. 1a of the original paper), while Nm sites accumulate at the $3^{\prime}$ ends (Supplementary Figs. 1 and 2; Supplementary Notes 1 and 2). However, because of the low Nm abundance in messenger RNA (mRNA), only very limited amounts of mRNA fragments carry $3^{\prime} \mathrm{Nm}$ and thus can be successfully ligated to the $3^{\prime}$ adaptor. Mispriming could occur if the $3^{\prime}$ end of the reverse transcription (RT) primer hybridizes to a few bases of the $5^{\prime}$-ligated RNA (Fig. 1a). Although our method effectively identifies Nm sites in abundant ribosomal RNA (rRNA, Supplementary Fig. 1), its application to less abundant mRNA can be contaminated by mispriming, leading to falsepositive Nm sites and the erroneous AGAUC motif on mRNA (original Fig. 2d), which corresponds to the $5^{\prime}$-end sequence of the 3 ' adaptor.

To eliminate mispriming, we kept the original procedure intact but designed new $3^{\prime}$ and $5^{\prime}$ adaptors with the following features (Fig. 1a): (i) we added a six-letter in-line barcode (ATCACG) at the $5^{\prime}$ end of the original $3^{\prime}$-adaptor sequence. After RT, all of the first-strand cDNAs generated from the correct priming should contain the complementary sequence of the in-line barcode. On the contrary, the cDNAs generated from mispriming will not contain it, as it will not be a part of the template to synthesize cDNA. We can thus readily identify and filter off the mispriming reads. (ii) We added 5-nt randomized nucleotides to the $3^{\prime}$ and 5' adaptors at the ligation junctions to reduce ligation-associated bias ${ }^{2-4}$. They also serve as unique molecular identifiers (UMIs) to identify and exclude PCR duplicates so that the real numbers of original molecules before PCR can be accurately quantified ${ }^{5}$.

With the elimination of mispriming, the refined Nm-seq was applied to the same input as in the original paper $(10 \mu \mathrm{g}$ mRNA from HeLa and HEK293 cells). Using a customized pipeline (see Online Methods), we detected 2,103 confident Nm sites from HeLa cells and $699 \mathrm{Nm}$ sites from HEK cells, respectively, with a $\mathrm{Nm}$ site distribution profile of Nm sites showing a similar distribution pattern as reported in the original Figure $2 c$ (Fig. 1b), and a different codon preference from the original Figure 2e (Fig. 1c, Supplementary Fig. 3, Supplementary Note 3). Additional features are summarized in Supplementary Figures $\mathbf{4}$ and 5, and consistent in HeLa and HEK293 cells (Supplementary Figs. 6 and 7 ). In both cell lines, Um is the dominant Nm modification ( $64 \%$ of all Nm sites in HeLa mRNA and $78 \%$ of all Nm site in HEK mRNA), which is consistent with our previous LC-MS/ MS data, with a depletion of A flanking the modification site (Fig. 1d). 